\title{
1 Title: Evidence for face selectivity in early vision
}

2 Classification: Biological Sciences (Psychological and Cognitive Sciences)

4 Authors: Florence Campana ${ }^{1}$, Jacob G. Martin ${ }^{1,2}$, Levan Bokeria ${ }^{1}$, Simon Thorpe ${ }^{2}$, Xiong Jiang ${ }^{1}$,

5 Maximilian Riesenhuber ${ }^{1 *}$.

\section{Affiliations:}

$7 \quad{ }^{1}$ Department of Neuroscience, Georgetown University Medical Center, Research Building, Room

8 WP-12, 3970 Reservoir Rd. NW, Washington, District of Columbia 20007, USA

$9{ }^{2}$ Centre de Recherche Cerveau \& Cognition, CNRS-Université Toulouse 3, 31059 Toulouse,

10 France

11

12 *Corresponding authors: mr287@georgetown.edu ; campana.florence@gmail.com

$14 \quad$ Number of pages : 52

15 Number of figures : 4

16 Number of words : Abstract (128), Significance Statement (119), Introduction (545), Discussion

$17(1415)$.

18

19 Conflict of Interest: The authors declare no competing financial interests. 
22 Acknowledgments: This research was supported by National Eye Institute Grant R01EY024161

23 and a grant from the Agence Nationale de la Recherche ANR-13-NEUC-004-01. We thank Dr.

24 Alex Todorov for face stimuli, and Drs. Bobby Stojanoski and Rhodri Cusack for scrambling code,

25 and Rebekah Farris for help with experiments.

26

27

28

29

30

31

32

33

34

35

36

37

38

39

40 


\section{Abstract}

42 The commonly accepted "simple-to-complex" model of visual processing in the brain posits that

43 visual tasks on complex objects such as faces are based on representations in high-level visual

44 areas. Yet, recent experimental data showing the visual system's ability to localize faces in natural

45 images within 100ms (Crouzet et al., 2010) challenge the prevalent hierarchical description of the

46 visual system, and instead suggest the hypothesis of face-selectivity in early visual areas. In the

47 present study, we tested this hypothesis with human participants in two eye tracking experiments,

48 an fMRI experiment and an EEG experiment. We found converging evidence for neural

49 representations selective for upright faces in V1/V2, with latencies starting around 40 ms post-

50 stimulus onset. Our findings suggest a revision of the standard "simple-to-complex" model of

51 hierarchical visual processing. 


\section{Significance statement}

53 Visual processing in the brain is classically described as a series of stages with increasingly

54 complex object representations: early visual areas encode simple visual features (such as oriented

55 bars), and high-level visual areas encode representations for complex objects (such as faces). In

56 the present study, we provide behavioral, fMRI, and EEG evidence for representations of complex

57 objects - namely faces - in early visual areas. Our results challenge the standard "simple-to-

58 complex" model of visual processing, suggesting that it needs to be revised to include neural

59 representations for faces at the lowest levels of the visual hierarchy. Such early object

60 representations would permit the rapid and precise localization of complex objects, as has

61 previously been reported for the object class of faces.

62

63 


\section{Introduction}

65 According to the standard model of visual processing (Hubel \& Wiesel, 1962 ; Maunsell \&

66 Newsome, 1987 ; Riesenhuber \& Poggio, 2002), simple physical features (e.g., luminance and

67 edges) are encoded first, in low-level visual areas such as V1, while more complex shape features

68 are encoded later in intermediate visual areas such as V4. Complex object categories (e.g., faces

69 and objects) are thought to be encoded in high-level visual areas such as the Fusiform Face Area

70 (FFA).

71 Humans are able to report the presence of specific objects (e.g. an animal or a vehicle) in natural

72 cluttered scenes as early as $250 \mathrm{~ms}$ post-stimulus onset ((Fabre-Thorpe et al., 2003; Poncet et al.,

73 2012; Thorpe et al., 1996; VanRullen and Thorpe, 2001). Those latencies are short, but compatible

74 with a progression of the neural activity from low-level to high-level visual areas, then followed

75 by the formation of a decision-variable in the frontal cortex (Freedman et al., 2003; Riesenhuber

$76 \&$ Poggio, 2000). However, the recent use of saccadic response paradigms, rather than manual

77 response paradigms, has revealed that object detection can be achieved substantially faster than

78 previously thought. Faces, for instance, can be saccaded to within $100 \mathrm{~ms}$ following stimulus onset

79 (Crouzet et al., 2010). Since conduction delays to generate an oculomotor response take around

$8020-35 \mathrm{~ms}$ (Heeman et al., 2017), this suggests that the visual detection has already been made by

$81 \quad 65-80 \mathrm{~ms}$ following stimulus onset. Such latencies pose a challenge for the standard hierarchical

82 view of the visual system. A second remarkable feature of these ultra-rapid saccades is their

83 localization accuracy: subjects are able to trigger saccades accurately toward very small faces of

$841^{\circ}$ visual angle pasted at an eccentricity of $7^{\circ}$ in a complex cluttered scene, after only $120 \mathrm{~ms}$

85 (Brilhault et al., 2011). V1/V2 neurons are a possible candidate since their receptive fields subtend 
$861^{\circ}$ in humans at $7^{\circ}$ eccentricity (Dumoulin \& Wandell, 2008), with the earliest responses starting

8745 ms post-stimulus onset (Foxe et al., 2008). By contrast, V4 receptive fields may be too large

88 since they subtend $4^{\circ}-6^{\circ}$ at $6^{\circ}$ eccentricity in humans (Motter 2009).

89 The precision of those saccades in cluttered environments and the fact that they start as early as

90 "express saccades" - the fastest known saccades in humans, that are directed toward luminous dots

91 (Fischer et al., 1984) - suggest that early visual areas, which are characterized by small receptive

92 fields and short activation latencies, might contain representations of complex object categories

93 such as faces.

94 In the present study, we tested the hypothesis of face-selective representation in early visual areas

95 using a multi-pronged approach. Specifically, to probe the temporal characteristics of putative

96 early face-specific responses, we conducted two eye-tracking experiments and an EEG

97 experiment. To probe the spatial characteristics of putative early face-specific responses, we

98 conducted a functional Magnetic Resonance Imaging (fMRI) experiment. In order to link the

99 results across experiments, stimuli were similar across experimental techniques. They consisted in

100 small faces of $2^{\circ}$ size, to match the size of V1/V2 receptive fields. We here report converging

101 evidence that faces elicit specific responses in V1/V2 as early as $40 \mathrm{~ms}$ post-stimulus onset. This

102 finding suggests a more nuanced picture of visual hierarchical processing than the traditional

103 "simple-to-complex" model, namely one in which early areas contains neuronal representations

104 selective for complex objects. 
106 Material and Methods

107 Across all studies, all subjects were naïve as to the purpose of the study, gave their written

108 informed consent before participating, and were paid for their participation. The Institutional

109 Review Board of Georgetown University approved the study.

110 Experiment 1: Specificity of ultra-rapid face detection

111 Experimental Design

112 Participants. Eight (6 women, $22.3 \pm 1.9$ years old across all subjects) healthy, right-handed

113 human subjects with normal or corrected-to-normal vision took part in the experiment.

115 Stimuli. The stimuli used in the experiment were natural scenes $\left(\mathrm{n}=15\right.$, resolution $15^{\circ} \times 20^{\circ}$ of

116 visual angle, from an online database) in which items (faces and distractors) were pasted. To avoid

117 that pasted items pop-out, the contrast histogram of the pasted item was stretched so that that the

118 bottom $1 \%$ and top $1 \%$ from the pixels of this item match the bottom $1 \%$ and top $1 \%$ from the

119 pixel values of the patch of natural scene replaced. All the images were grayscale.

120 Items were pasted at one of four locations at $7^{\circ}$ visual eccentricity from the center of the screen:

12125 or $45^{\circ}$ above and below the horizontal meridian, on the left and right side of the screen. This

122 asymmetrical spatial configuration aimed at activating the primary visual cortex at symmetrical

123 sites on the lower and upper banks of the calcarine fissure (Di Russo et al., 2005) granted that the

124 horizontal meridian is represented in the lower bank of the calcarine fissure (Aine et al., 1996). To

125 approximately match the size of the receptive fields in V1/V2 at $7^{\circ}$ eccentricity (Dumoulin \&

126 Wandell, 2008), all faces and distractors were resized to span $2^{\circ}$ of visual angle (along the height

127 dimension). $7^{\circ}$ eccentricity was chosen to be in the range of the eccentricity used in (Brilhault et 
128 al., 2011), in which fast saccades toward faces were observed. 15 emotionally neutral faces of

129 males generated with the FaceGen 3.1 software development kit (Singular Inversions, Toronto,

130 Canada) were used. Elements external to the faces (necks and shoulders) were manually removed.

131 From those faces, new face images (inverted, configuration-scrambled and phase-scrambled faces)

132 were created using the Gimp image manipulation program (https://www.gimp.org/) and MATLAB

133 (The Mathworks, MA): "configuration-scrambled" faces in which the internal features (the eyes,

134 the nose and mouth) were positioned at random places, inverted faces, and phase-scrambled face

135 (Xu et al., 2005). 15 images of houses (online database) were also used as distractors (Figure 1A).

136 In this experiment, the "configuration-scrambled" faces, the inverted faces, the phase-scrambled

137 faces and the houses are called "distractors".

Procedure. Stimuli were presented on a computer screen at a viewing distance of $60 \mathrm{~cm}$ (refresh

140 rate $60 \mathrm{~Hz}$, resolution $1024 * 768,24 * 18^{\circ}$ visual angle). Eye movements were recorded using a

141 camera-based eye tracker (SR research, Eyelink 1000 Plus) with a temporal resolution of $2000 \mathrm{~Hz}$.

142 A chin and headrest helped the subjects to stabilize their head. Each trial started with a white

143 fixation cross. Subjects had to keep their eyes on the cross (within a box of radius $1^{\circ}$ of visual

144 angle) for $150 \mathrm{~ms}$ for the natural scene to be displayed. A 200-ms gray screen was displayed

145 between the end of the fixation and the start of the natural image. This gap enables a faster initiation

146 of saccades (Fischer \& Weber, 1993, Kirchner \& Thorpe, 2006, Crouzet et al., 2010). Subjects

147 were instructed to saccade as quickly as possible toward the face pasted in the natural scene. The 148 natural scene was displayed until the subject's eyes gazed at the face for 150 consecutive ms in a 149 square zone centered on the face and of $2^{\circ}$ width. Next fixation started after a pseudo-random 150 interval (1000-1200 ms). Subjects performed 15 blocks of 72 trials each. Within a block, there 
151 were 48 trials with a face and a distractor (12 possible spatial configurations, one per distractor

152 category), 12 trials with a face only (4 positions, each repeated 3 times), and 12 trials with two

153 faces presented simultaneously (6 possible spatial configurations, each repeated twice). To assess

154 the effect of the different distractor categories independently of face identity, the identity of the

155 face and of the distractor (inverted, scramble or configuration-scrambled) were always the same

156 within an image. Each of the 15 backgrounds and each of the 15 face identities were displayed 72

157 times (pseudo-random association of a face with a background).

158 Subjects were informed that, in any trial, the faces would appear at 1 out of 4 possible locations

159 (left up, left bottom, right up, right bottom) (Figure 1A). They were informed that the images

160 would contain distractors, some of them sharing similarities with faces, but that only the "normal

161 faces" were relevant for the task.

\section{Statistical Analyses}

164 The position of the left eye from each participant was recorded during the whole experiment,

165 every half ms. Only the first saccade within each trial was kept in the analysis. For each

166 distractor condition, we computed the percentage of saccades landing in the quadrant with a face,

167 in the quadrant with a distractor, and in empty quadrants. This measure was averaged across

168 subjects. We also computed saccadic reaction times, measured as the duration elapsed between

169 the display of the stimulus and the initiation of the saccade, as recorded by the eye tracker. This

170 measure was averaged across subjects too. We computed the minimum saccadic reaction times

171 (SRT), which is a statistical estimate of the latency at which correct saccades start to be more

172 numerous than saccades toward the distractor's quadrant (Crouzet et al., 2010). For each subject,

173 we computed the cumulative count of number of saccades whose SRT was inferior to a cutoff 
174 value, step size $1 \mathrm{~ms}$. We then searched for at least 20 consecutive bins with significantly more

175 correct than erroneous responses using a chi-square test with a criterion of $\mathrm{p}<0.05$. The first of

176 those bins was considered to correspond to the minimum SRT.

177 Experiment 2: Timing and spatial accuracy of ultra-rapid face detection

178 Experimental design

179 Participants. Sixteen (14 women, $23.6 \pm 4.1$ years old across all subjects) healthy, right-handed

180 human subjects with normal or corrected-to-normal vision took part in the experiment.

181 Stimuli. Stimuli consisted of annuli (radius: $7^{\circ}$ of visual angle, width: $2.5^{\circ}$ of visual angle) cut out

182 from the same database of natural scenes as used in Experiment 1. In each trial, a face (height: $2^{\circ}$

183 of visual angle) was pasted at a random polar angle, always at $7^{\circ}$ eccentricity from the center of

184 the screen (Figure 2A). 48 grayscale scenes with a high degree of clutter were selected after visual

185 inspection to make sure that, after the procedure of luminance equalization described above, the

186 pasted faces were still clearly visible at any position within at least 2 out of 4 quadrants in the

187 annulus. 36 grayscale photographs of adult faces of various ethnicities were used (online database)

188 to maximize the ecological value of our results. The neck and shoulders were manually removed

189 from those images as in Experiment 1. Eye movements were recorded using the same setup as in

190 Experiment 1.

191

192 Procedure. The experimental setup was identical to that of Experiment 1 except that the distance

193 between the subjects' eyes and the screen was $93 \mathrm{~cm}(60 \mathrm{~cm}$ for the two first subjects, but stimulus

194 size in degrees of visual angle was identical). 36 faces were displayed, and subjects were instructed

195 to saccade as quickly as possible to the faces. Similarly to Experiment 1, once the participant's 196 gaze was within the face for 150 consecutive $\mathrm{ms}$, the next trial started after a pseudo-random 
197 interval (between 1000 and $1200 \mathrm{~ms}$ ). Each face was displayed once within each quadrant $(36 * 4$

$198=144$ trials).

\section{Statistical Analyses}

201 To assess the properties of the earliest saccades that can be selectively oriented toward faces, only

202 the first saccade of each trial was kept for analysis. We computed the minimum saccadic reaction

203 times (SRT) with the same method as described in Experiment 1.

205 Experiment 3: EEG

206 Experimental Design

207 Participants. 21 healthy (9 women, $24 \pm 4.2$ years old across old subjects), right-handed human

208 subjects with normal or corrected-to-normal vision took part in the experiment. 2 subjects out of

20921 were removed from the group analysis (one since the image was removed in $30.6 \%$ of the trials

210 due to eye movements, the other due to the small amplitude of his N170 upright-face evoked

211 component, at more than 3 std from the mean (average amplitude of the peak, left-presentation

212 group, left-hemisphere: $-4.3 \pm 1 \mu \mathrm{V}$, individual value for this participant: $-0.49 \mu \mathrm{V}$; on the right

213 hemisphere: $-4.7 \pm 1 \mu \mathrm{V}$, individual value: $-1.7 \mu \mathrm{V})$.

214 Stimuli. 100 different grayscale pictures of faces (the same as in the Experiment 2, plus 64 from

215 the same database, from which the neck and shoulders were removed) were used in this

216 experiment.

218 Procedure. The eyetracking setup was identical to that of Experiment 1 . In 10 out of the 19 subjects

219 included in the group analysis, eye movements were tracked with the Eyelink SR 1000 (for the 
220 other subjects, eye movements could not be recorded because of difficulties with calibrating the

221 eye tracker due to glasses and/or contact reflection). Scalp voltages were measured using an

222 Electrical Geodesics (EGI, Eugene, OR) 128-channel Hydrocel geodesic sensor net and Net Amps

223300 amplifier. Incoming data were digitally low-pass filtered at $200 \mathrm{~Hz}$ and sampled at $500 \mathrm{~Hz}$

224 using common mode rejection with vertex reference. Impedances were set below $75 \mathrm{k} \Omega$ before

225 recording began and maintained below this threshold throughout the recording session with regular

226 impedance checks between blocks.

228 Half of the subjects $(n=9)$ were presented with faces in the left visual field, among which 5 had

229 their eye movements recorded. The other half was presented with faces in the right visual field (n

$230=10$ ), among which 5 had their eye movements recorded. Grayscale pictures of faces were

231 displayed on a gray background on the horizontal meridian. We chose a gray background rather

232 than a natural one to avoid a reduction of the amplitude of face-related signals (Cauchoix et al.,

233 2014). To engage attention to the faces, as in Rossion \& Caharel, 2011, we engaged subjects in a

234 face categorization task (upright vs. inverted). The faces were either displayed at $1^{\circ}$ eccentricity

235 (height: $0.93^{\circ}$ visual angle) or at $7^{\circ}$ eccentricity (height: $2^{\circ}$ visual angle), with eccentricity chosen

236 randomly for each trial (50 faces at $1^{\circ}$ eccentricity, 50 faces at $7^{\circ}$ eccentricity). The faces size was

237 determined using the cortical magnification factor in V1 whose value was computed from the

238 formula in (Rousselet et al., 2005) so that, at the two presentation eccentricities, the faces stimulate

239 similar portions of V1. Each trial began with a fixation point displayed for 300-600 ms followed

240 by the brief display of a face, either upright or inverted, for $150 \mathrm{~ms}$. $150 \mathrm{~ms}$ was chosen as it is

241 longer than our time window of interest $(\sim 50-100 \mathrm{~ms})$ to avoid contamination of possible face-

242 selective signals by stimulus-offset transient responses caused by the stimulus disappearance 
243 (Macknik \& Livingstone, 1998). Accurate stimulus timing was verified with a photodiode. The

244 subjects then reported whether the face was upright or inverted by pressing the ' 1 ' or ' 3 ' key,

245 respectively, with their right index and ring fingers (timeout: 2 seconds). After $600 \mathrm{ms,}$ a new trial

246 began. Subjects were asked to keep their eyes on the fixation point during the overall duration of

247 a trial. Subjects were instructed to withhold eye blinks until the end of a trial and could pause the

248 experiment between trials to blink and/or rest. For subjects whose eye movements were recorded,

249 the face appeared only after 150 consecutive ms with the eyes on the fixation spot, thus extending

250 the duration of the fixation in certain trials. For those subjects, the face was removed and the trial

251 aborted if the subject was moving his/her eyes outside of the fixation point during the face

252 presentation. The configuration (upright or inverted), the eccentricity and the identity of faces were

253 pseudo-randomized and counterbalanced across the experiment. Subjects performed 10 blocks of

254100 trials each.

255 Statistical Analyses

256 Data Analysis. Data pre-processing and statistical analyses were performed using EEGLAB

257 (Delorme \& Makeig, 2004), FieldTrip (Oostenveld et al., 2011) and MATLAB (The Mathworks,

258 MA). Noisy channels were interpolated. Data were band-pass filtered between 0.1 and $30 \mathrm{~Hz}$ with

259 a causal filter. A causal filter was used in order to avoid shifts in the latency of the ERPs (Rousselet,

260 2012). The filtered signal was then epoched from $150 \mathrm{~ms}$ before stimulus to $250 \mathrm{~ms}$ after the

261 stimulus, baseline corrected in the $[-150,0] \mathrm{ms}$ time window, and trials in which one of the facial

262 channels was exhibiting a variation of more than $75 \mu \mathrm{V}$ in the $[-150,+200]$ ms time window were

263 removed. Only trials in which the response was correct were kept. Event-related potentials (ERPs)

264 were averaged separately for each subject and for each face orientation (upright, inverted). ERP

265 subject averages were then grand-averaged separately across the subjects with left-face 
presentation, and across the subjects with right-face presentation. Significant differences between

267 the ERP amplitude in the upright versus inverted conditions were detected (separately within the

268 left-presentation group and the right-presentation groups) using paired t-tests (one-tailed, threshold

269 at 0.05 , to test the hypothesis that upright faces elicit a signal of larger amplitude than inverted

270 faces) in a clustering procedure (Oostenveld et al., 2011), with a minimum cluster size threshold

271 of 5 (Scholl et al., 2014). This clustering analysis was performed on all the trials, combining both

272 eccentricity conditions to increase the signal-to-noise ratio. We ran the analysis on the [0-150ms

273 time window], i.e., during the stimulus presentation. Signals were selected over the most posterior

274 channels $(n=51)$, that broadly overlaid posterior visual areas.

\section{Experiment 4: fMRI}

276 Participants. 16 healthy, right-handed human subjects (5 women, $20.54 \pm 1.1$ years old) with

277 normal or corrected-to-normal vision took part in the experiment. Three subjects were excluded

278 from the group analysis: one was singing in the scanner and unable able to focus, the other

279 turned out to be under medication (Truvada), the third one had an excessive amount of head

$280 \operatorname{motion}(>8 \mathrm{~mm})$.

\section{Experimental design}

282 Procedure. In the main experiment, stimuli consisted of natural grayscale scenes (the same as in

283 the experiments 1 and 2, 50 different scenes) on which 4 items were pasted (height $2^{\circ}$, at $7^{\circ}$

284 eccentricity, at 25 and $45^{\circ}$ above and below the horizontal meridian), to match the location and

285 size of the items in Experiment 1. The items were pasted on gray circles of $4.5^{\circ}$ diameter to reduce

286 the effect of variations in the scene content on the BOLD response. Within an image, the four

287 items consisted of an upright item (U), the inverted version of $U(I)$, the scrambled version of $U$ 
288 (US), the inverted version of US (IS). U was either a face (50 different photograph of faces, chosen 289 from the same set as in Experiments 2 and 3), or a house (25 different photographs of houses, from 290 an online database) (Figure 4A). The arrangement of the items U, I, IS, US on the four gray circles

291 (top left, top right, bottom left, bottom right) was varied across trials, thereby defining four 292 configurations. The scrambling procedure to create the scrambled items was the one introduced by 293 Stojanoski \& Cusack, 2014, which keeps low-level properties of the items: a degree of warping of 29435 was used in order to make the face category unrecognizable. Each participant viewed a total of 295 four blocks (125 trials per block, 25 faces * 4 spatial configurations, plus 25 houses). The sets of 296 faces and houses were repeated twice during the experiment, between two blocks, randomized 297 between subjects. Within one block, the 25 house trials had the same spatial configuration, with a 298 different spatial configuration between each block.

Main experiment. The purpose of the experimental design in this experiment was to mask the 301 faces and made them invisible to the participants. Each trial (Figure 4B) started with a white 302 fixation cross on a gray background that was displayed for $1000 \mathrm{~ms}$ and was followed by a stream 303 of 12 characters pseudo-randomly chosen from a set containing the letters of the alphabet, the 304 numbers $1-9$, and 10 punctuation marks $(\{!, @, \#, \$, \%, \wedge, \&, *,()\}$,$) , and presented in a rapid$ 305 sequence (5 frames each, 60Hz refresh rate) (adapted from (Beck \& Kastner, 2005)). In each trial, 306 a natural scence (with face items or house items, as described above) was embedded in the stream 307 and stayed on the screen for 4 frames. In $70 \%$ of the trials, there was an ' $L$ ' in the stream. Subjects 308 were instructed to press a button as soon as they saw the letter ' $L$ '. The rapid serial visual 309 presentation aimed at keeping the attention maximally engaged on the center of the screen. The 310 letter 'L' was always displayed after the natural scene so that subjects were fully focused on the 
311 letter stream at the moment of appearance of the natural scene. The precise timing of the

312 presentation of the stimuli of interest (the natural scene with four faces/four houses) was chosen

313 randomly, it occurred between the 4th to 8th character and could thus not be predicted. Subjects

314 were instructed to keep their gaze and attention on the stream of characters in the middle of the

315 screen. They were told that the natural scenes displayed were irrelevant to the task.

317 MRI acquisition parameters. Data were acquired with a 3T MRI scanner (Magnetom Trio,

318 Siemens) at Georgetown University's Center for Functional and Molecular Imaging. A 12-channel

319 head coil was used, $\mathrm{TR}=2040 \mathrm{~ms}, \mathrm{TE}=29 \mathrm{~ms}$, flip angle $=90^{\circ}, 35$ interleaved axial $($ thickness $=$

$3204.0 \mathrm{~mm}$, no gap; in-plane resolution $=3.2 \times 3.2 \mathrm{~mm}^{2}$ ). At the end, 3D T1-weighted MPRAGE

321 images (resolution $1 \times 1 \times 1 \mathrm{~mm}^{3}$ ) were acquired from each participant. Visual stimuli were back-

322 projected from a computer screen (resolution: 768*1024) on a mirror within the scanner (distance

323 mirror-eyes: $89 \mathrm{~cm}$ ). Data from four runs of event-related scans, one run of face localizer scan,

324 and two runs of retinotopic localizer scans were acquired from each participant.

326 V1 retinotopic localizer scans. The V1 localizer scans aimed at identifying the portions of V1 (4)

327 activated by presentation of the faces and houses, in order to focus our analyses on those regions.

328 High-contrast achromatic flickering (contrast-reversing at $20 \mathrm{~Hz}$ ) checkerboard patterns were

329 displayed on a gray background (diameter, $3^{\circ}$ ) successively at the 4 positions occupied by the

330 pasted items (top: left and right; bottom: left and right, at $7^{\circ}$ eccentricity). The checkerboards were

331 displayed for 16 consecutive secs at each position and each position was repeated twice. During

332 the presentation of the checkerboards, in the middle of the screen, the letters ' $\mathrm{T}$ ' and ' $\mathrm{L}$ ' were

333 displayed in alternation, in a randomized order (50 ms of presentation per letter, frequency of 
334 occurrence of the letter L: 70\%). Similar to the main experiment, subjects were instructed to press

335 a button every time they were seeing the letter ' $L$ '. The first retinotopic localizer scan was run

336 right before the main experiment, the other scan was run right after the main experiment.

338 Face localizer scan. After the second retinotopic localizer scan, a face localizer scan was run to

339 identify the Fusiform Face Area (FFA) in each subject. We used stimuli and a similar design as

340 described in (Kanwisher et al., 1997): 50 different grayscale images of faces and houses, distinct

341 from the ones in the main experiment, were displayed in the center of the screen in blocks on a

342 uniform background. Each block, either with faces or with houses, was run twice. The face and

343 house images were purchased from a commercial source and post-processed using programs

344 written in MATLAB (The Mathworks, MA) to eliminate background and to adjust image size (to

$345200 * 200$ pixels), luminance and contrast. 10 subjects were instructed to passively view the

346 stimuli, 3 others were engaged in a 1-back task to increase attentional engagement.

\section{Statistical Analyses}

348 Behavioral Data Analysis. We computed the signal detection theoretic (SDT) measures d' and c

349 (MacMillan \& Creelman, 2005) in the target letter detection task. The variable d' is a measure of

350 a participant stimulus discrimination sensitivity (here, between the letter ' $\mathrm{L}$ ' and other symbols),

351 while $\mathrm{c}$ is a measure of a participant bias to report the letter ' $\mathrm{L}$ '. These measures were calculated

352 on the basis of the rate of hits (letter ' $L$ ' reported in trials in which the letter ' $L$ ' was present) and

353 false alarms (letter ' $L$ ' reported in trials in which the letter ' $L$ ' was absent).

355 MRI Data Analysis. All processing and most statistical analyses were done using the SPM8 356 software package (http://www.fil.ion.ucl.ac.uk/spm/software/spm8/). The first five volumes of 
each scan were discarded to allow for scanner equilibration. The functional scans and the localizer scans were preprocessed separately. The echoplanar images (EPIs) were reoriented, then

Neurological Institute (MNI) template brain. Images were then smoothed with a full-width at halfpass filter $(1 / 128 \mathrm{~Hz})$, fMRI responses were modeled with a design matrix comprising the onset

364 function.

Identification of V1 Regions of interest (ROIs). V1 regions of interest were identified from the

367 two retinotopic localizer scans, separately for each participant, via the MarsBar toolbox (Brett et

versus the three other checkerboard positions and this was performed for each of the 4 positions.

372 presented in the lower visual field. We could infer from (Dougherty et al., 2003) that each

373 checkboard (diameter: $3^{\circ}$, at $7^{\circ}$ eccentricity) should activate around $75 \mathrm{~mm}^{3}$ of cortical surface in

374 V1, i.e. approximately 10 voxels of $8 \mathrm{~mm}^{3}$. Based on this estimation, to define V1 ROI, we

375 adjusted the statistical threshold to obtain, in each participant, clusters of approximately 10-15

376 voxels (significant at the corrected cluster level of at least $\mathrm{p}<0.05$, else, at the uncorrected cluster

377 level of at least $\mathrm{p}<0.001)$ (Glezer et al., 2015, Jiang et al., 2006). We thus aimed at identifying

378 V1 ROIs, through the use of checkerboard stimuli which are known to strongly activate V1 (Engel

379 et al., 1997), and by restricting the ROIs to 15 voxels. However, for reasons of parsimony, because 
380 the activity in V1 propagates to $\mathrm{V} 2$, we interpret our data as being in V1/V2, in other words in the

381 early visual cortex.

383 Identification of face-selective ROIs. Face-selective ROIs were identified from the localizer scan

384 separately for each participant, via the MarsBar toolbox (Brett et al., 2002). Epochs with face and

385 house stimuli were modeled with two box-car functions convolved with a canonical hemodynamic

386 response function (HRF). Participant-specific ROIs were defined by voxels that displayed face $>$

387 house responses. We focused our analysis on the right FFA (Kanwisher et al., 1997, Jiang et al.,

388 2006). ROIs were selected by identifying in each participant the cluster in the right temporal cortex

389 that was significant at the cluster level (corrected cluster level, at least $\mathrm{p}<0.05$ else, uncorrected

390 cluster level, at least $\mathrm{p}<0.001)$. With such thresholds, only subjects for whom a cluster of at least

39130 voxels was found, in a location close to the published location of the right FFA, approximate

392 MNI coordinates (39 $\pm 3-40 \pm 7-16 \pm 5)$ (Grill-Spector et al., 2002) were included in the FFA

393 analysis ( $\mathrm{n}=8$ out of 13). In 3 out of the 5 subjects for which no right FFA-cluster could be found

394 with the contrast face $>$ house, we could obtain, through the contrast face $>$ baseline, a right FFA-

395 cluster in a location close to the published location of the right FFA according to Grill-Spector et

396 al., 2002. Analyses were run within the homogenous set of 8 subjects whose FFA was defined via

397 the contrast face $>$ house, and, to increase the statistical power, analyses were also run with the 3

398 additional subjects with the cluster found via the contrast face $>$ baseline. 


\section{Results}

\section{Experiment 1: Fast saccades toward faces have limited selectivity}

402 We asked subjects to saccade, as fast as they could, toward faces in natural scenes containing a

403 face as well as a distractor sharing, to different degrees, physical properties with faces.

404 The comparison of the percentage of saccades oriented toward the face versus the distractor 405 revealed that, when the distractor was a configuration-scrambled face (i.e., a face whose internal

406 parts had been scrambled randomly, see Figure 1 A), subjects made as many saccades toward the

407 configuration-scrambled face as toward the target face (Figure 1B), despite the instruction to

408 saccade toward the normal face (\% saccades in the quadrant with the normal face: $40.31 \% \pm$ $4091.72 \%$, with a configuration-scrambled face: $42.93 \% \pm 2 \%$, paired $t$ test: $\left.T_{(7)}=-1.18, p=0.27\right)$. In

410 trials with an inverted face, a phase-scrambled face or a house, subjects made more saccades

411 toward the face than toward the distractor (inverted face distractors: \% saccades in the quadrant

412 with the face: $49.28 \% \pm 2.1 \%$, with an inverted face: $34.63 \% \pm 1.67 \%, \mathrm{~T}_{(7)}=6.6, \mathrm{p}=3 * 10^{\wedge}-4$;

413 house distractors: \% saccades in the quadrant with the face: $68.35 \% \pm 3.35 \%$, house distractors:

$41414.9 \% \pm 0.7 \%, \mathrm{~T}_{(7)}=13.89, \mathrm{p}=2 * 10^{\wedge}-6$; phase-scrambled distractors: with the face: $65.92 \% \pm$

$4153.53 \%$, with a phase-scrambled face: $\left.16.06 \% \pm 1 \%, \mathrm{~T}_{(7)}=11.9, \mathrm{p}=6 * 10^{\wedge}-6\right)$. The min SRT, a

416 statistical estimate of the latency at which the first saccades to the (target) face start to be more

417 numerous than toward the distractor (Material and Methods), were, on average, $140+22 \mathrm{~ms}, 149$

$418 \pm 7 \mathrm{~ms}, 136 \pm 11 \mathrm{~ms}, 138 \pm 15 \mathrm{~ms}$ (in the configuration-scrambled, inverted face, phase-scrambled,

419 house distractor conditions, resp.). Note that minimum SRTs in this experiment were longer than

420 in Crouzet et al., 2010, presumably since subjects expected the distractors to be similar to the target

421 and therefore potentially adopted a more cautious strategy. 
423 Since saccades to faces have been found to be more accurate and faster when the faces were

424 displayed in the left visual field rather than in the right visual field (Crouzet et al., 2010), we

425 conducted a separate analysis of the saccades when the face and the distractor were both in the left

426 hemifield versus both in the right hemifield. In trials with an inverted face distractor, a higher

427 number of saccades toward the face relative to the distractor was observed for left-hemifield

428 presentation of the face and distractor (\% saccades within the quadrant with the face: $51 \% \pm 1 \%$,

429 with an inverted face: $\left.38.6 \% \pm 1 \%, \mathrm{~T}_{(7)}=5.24, \mathrm{p}=0.001\right)$. In contrast, for right-hemifield

430 presentation of the face and distractor, subjects made saccades as often toward the inverted face as

431 toward the upright target face (\% saccades within the quadrant with the face: $41 \%$, with an inverted

432 face: $36 \%$, paired t test: $\mathrm{T}_{(7)}=0.84, \mathrm{p}=0.42$ ) (Figure $\left.1 \mathrm{C}\right)$, suggesting lower face selectivity for

433 right-hemifield presentations. For the other categories, we did not expect such an asymmetry

434 because configuration-scrambled faces act as perfect distractors while houses and phase-scrambled

435 faces do not act as effective distractors.

437 In summary, Experiment 1 provided evidence that fast face detection has limited shape selectivity, 438 with configuration-scrambled faces frequently being mistaken for targets. This finding is 439 compatible with the notion of fast face detection being based on detectors that encode only face 440 parts, not holistic faces. Supporting this hypothesis, inverted faces, whose parts are affected 441 somewhat by inversion, were not as effective distractors as the configuration-scrambled faces. 442 Another interesting finding from Experiment 1 was a hemispheric asymmetry of fast face 443 detection, with an advantage for face detection in the left visual hemifield. We next conducted 
444 another experiment to more finely assess the spatial precision as well as lateralization effects of

445 the earliest saccades oriented toward faces.

446 Experiment 2: Faces are saccaded to faster and more precisely when they are in the

447 left hemifield

448 In Experiment 2, subjects were asked to detect faces in "annulized" natural images consisting of a

449 natural scene windowed by an annulus, which contained a face image ( $2^{\circ}$ of visual angle high).

450 The saccades started as early as $100-120 \mathrm{~ms}$ post stimulus onset (Figure $2 \mathrm{C}$ ). Figure $2 \mathrm{~B}$ shows that

451 saccades were aggregated in an area centered on the faces and extending up to about 15-20 polar

452 degrees on both sides. We therefore labeled as "correct" the saccades that landed in an area

453 centered on the faces and extended up to 15 degrees both sides and computed the minSRT

454 (Material and Methods). The correct saccades represented $59.6 \pm 4 \%$ of the total number of 455 saccades and landed at $1.07^{\circ} \pm 0.05^{\circ}$ visual angle from the center of the face, with an average 456 latency of $182.3 \mathrm{~ms}$ (average of the moment of onset of the first saccades). The minimum SRT 457 (Material and Methods) was shorter for left presentations than for right presentations (minimal 458 SRT, on average, left-presentation: $160 \pm 4 \mathrm{~ms}$, right presentation: $177 \pm 4 \mathrm{~ms}$, paired t-test, $\mathrm{T}_{(15)}$ $459=-3.2, p=0.008$ ) (Figure 2C). Furthermore, saccades landed closer to the face in left-presentation 460 trials relatively to right-presentation trials (left presentation: $3.54^{\circ} \pm 0.28^{\circ}$, right presentation: 4.06 $461{ }^{\circ} \pm 0.25^{\circ}$, paired t-test: $\left.\mathrm{T}_{(15)}=-2.8, \mathrm{p}=0.013\right)$. 
466 To probe the neural bases of fast face localization, we conducted a high-density

467 electroencephalography (EEG) study and assessed the earliest latency of face-selective neural

468 signals. We presented faces in the periphery while asking subjects to look at a central fixation spot

469 and perform an upright/inverted face classification task on peripherally presented faces. They were

470 presented on one side of the screen only, with presentation side alternating between subjects. Akin

471 to previous studies of higher-level face processing (Kanwisher et al., 1998 ; Rossion et al., 2000),

472 and motivated by the observation of an advantage for upright vs. inverted faces in fast face

473 detection (Experiment 1), we adopted a stringent test to establish face selectivity of neural

474 responses, requiring significantly different neural responses to upright vs. inverted faces. This

475 contrast avoids confounds arising from low-level stimulus differences between faces and

476 comparison objects (e.g., when comparing responses to faces versus houses, which can be

477 differentiated by other low-level features such as different luminance distributions). To engage

478 attention to the faces, as in (Rossion \& Caharel, 2011), we engaged subjects in a face categorization

479 task (upright vs. inverted).

480 Reaction times in the upright/inverted face categorization tasks were computed on the trials kept

481 for the EEG analysis, i.e., on the correct trials. Across the 10 subjects whose eye movements were

482 recorded, $11.7 \pm 2.8 \%$ of the trials on average were aborted due to eye movements. Subjects were

483 faster responding to upright faces than inverted faces (paired t-test, mean average reaction time for

484 correct trials, left-presentation group, upright faces: $538 \pm 28.3 \mathrm{~ms}$, inverted faces: $586 \pm 35 \mathrm{~ms}$,

$485 \mathrm{~T}_{(8)}=-3.6, \mathrm{p}=0.0069$; right-presentation group, upright faces: $603 \pm 48 \mathrm{~ms}$, inverted faces: $651 \pm$

$48648.5 \mathrm{~ms}, \mathrm{~T}_{(9)}=-4.4, \mathrm{p}=0.0016$; note that these reaction times were substantially longer than the 
$487 \quad 0-100 \mathrm{~ms}$ time window of interest). Reaction times were not significantly different between the 488 left- and right-presentation groups (unpaired t-test: $\mathrm{T}_{(18)}=-1.05, \mathrm{p}=0.3$ ). Accuracy was very high, 489 and similar between the groups (unpaired t-test, mean accuracy, left-presentation group: $93.3 \pm$ $4901 \%$, right-presentation group: $91.3 \pm 2.5 \%, \mathrm{~T}_{(18)}=0.72, \mathrm{p}=0.47$ ). The average number of trials 491 kept for the EEG analysis was similar in the left- and in the right-presentation groups (unpaired t492 test, left-presentation group: $705 \pm 43$ trials, right-presentation group: $625 \pm 27$ trials, $\mathrm{T}_{(18)}=1.6$, $493 \mathrm{p}>0.1)$

495 A clustering analysis was done using Fieldtrip (Oostenveld et al., 2011) between 0-150 ms 496 relatively to stimulus onset over the most posterior channels, which capture (early) visual-evoked 497 responses and notably V1 activity (Foxe \& Simpson, 2002). In the left-presentation group, a 498 significant cluster $(\mathrm{p}=0.035)$ differentiating the signal in the upright and inverted conditions 499 between 40 and $110 \mathrm{~ms}$ was found (Figure 3A). The cluster was lateralized in the right hemisphere 500 (Figure 3B). In the right-presentation group, the cluster started later, extending from 98 to $140 \mathrm{~ms}$ $501(\mathrm{p}=0.04)$ (Figure 3C). The cluster was initially lateralized in the left hemisphere (Figure 3D). A 502 control analysis was run to verify that the early clusters with a significantly higher signal amplitude 503 for upright vs. inverted faces were not driven by subjects without eye recordings, who could have 504 moved their eyes despite the instruction not to do so. Specifically, we computed the mean signal 505 amplitude for each condition (upright and inverted configurations) within the subjects whose eye 506 movements were recorded. We predicted that for participants whose eye movements were 507 recorded and who maintained fixation throughout each trial (Material and Methods), thus with no 508 possible contamination of the ERP by eye movements, we should observe a significant difference 509 between the upright vs. inverted conditions in the ERP. In this analysis, we combined the 
510 participants with left-hemifield and right-hemifield presentation in order to increase statistical

511 power. This analysis revealed that the differences were indeed observed within those subjects

512 (group of subjects with eye movements recorded, within the appropriate cluster according to the

513 presentation side, upright condition: $1.16 \pm 0.36$, inverted condition: $0.87 \pm 0.32, \mathrm{~T}_{(10)}=3.9, \mathrm{p}=$

514 0.0029). This confirms that the early face selectivity could not be explained by differential

515 movements toward upright versus inverted faces.

518 To identify the sources of the early face-selective neural signal, we conducted an fMRI study.

519 Subjects were engaged in a central letter detection task during a rapid serial visual presentation

520 (RSVP) of symbols and letters, while small faces and houses $\left(2^{\circ}\right)$ were displayed in the periphery

$521\left(7^{\circ}\right)$. In the central letter detection task, subjects had, on average, a high d-prime that stayed stable

522 across blocks, thus suggesting that they stayed engaged over the whole duration of the experiment

523 (across the four blocks, mean, d' $=3.3$, mean criterion c: -0.8 ; repeated-measures ANOVA over

524 criterion $\mathrm{c}$ with the single factor Block, $\left.\mathrm{F}_{(1,12)}=2.05 \mathrm{p}=0.12\right)$. Debriefing at the end of the

525 experiment revealed that none of the subjects noticed either faces or houses items.

527 We used upright faces (U), inverted faces (I), scrambled upright (US) and scrambled inverted (IS)

528 faces in order to probe for face selectivity in V1. Similarly to the EEG Experiment (Experiment

529 3), we used inverted faces to assess face selectivity. We also used scrambled faces as a control.

530 The scrambling procedure (from Stojanoski \& Cusack, 2014, described in the Methods section)

531 preserved the faces' basic visual properties, to which the earliest stages of the visual processing 
532 are sensitive, while making faces unrecognizable as faces. We reasoned as follows: if there is some

533 selectivity to faces in V1, the BOLD signal in response to $\mathrm{U}$ faces should be higher than for the

534 other three categories. The contrast \{US vs. IS \} enabled us to check that a differential BOLD

535 signal between $U$ versus I items does not arise from an inversion effect but is truly a response

536 specific to upright faces.

538 Retinotopic localizer scans with flickering checkerboards known to evoke strong responses in V1

539 (Engel et al., 1997) were used to define the regions of V1 activated during the functional runs by 540 our visual stimulation in periphery ( 4 items pasted at $7^{\circ}$ eccentricity, see Figure 4A). The four

541 ROIs were in the lower and upper bank of the left and right calcarine sulcus, corresponding to the

542 upper and lower presentation of the checkerboards respectively (Material and Methods, mean

543 coordinates of the ROIs: activated by top left presentations: $(12 \pm 1-78 \pm 1-5 \pm 1)$, bottom left:

544 (12 $\pm 1.1-92 \pm 121 \pm 2)$, top right: $(-10 \pm 1-81 \pm 1.3-8 \pm 1.2)$, bottom right: $(-8 \pm 1-97 \pm 116 \pm$

545 2)). The ROI subtended approximately 15 voxels (mean size of the ROI: $13.9 \pm 0.68$, activated by

546 top left presentations: $13.07 \pm 0.67$, bottom left presentations: $12.08 \pm 0.53$, top right presentations:

$54714.2 \pm 0.94$, bottom right presentations: $16.15 \pm 2.5$, repeated-measures ANOVA over the ROIs

548 size, factor ROI location: $\left.\mathrm{F}_{(1,12)}=0.13, \mathrm{p}=0.25\right)$.

550 We first considered the percent signal change collapsed across the four ROIs. Responses in V1/V2

551 to upright and inverted faces were similar, regardless of scrambling status: the unscrambled faces

552 (mean \% signal change, U: $0.34 \pm 0.04$, I: $0.33 \pm 0.04$, paired t-test, $\mathrm{T}_{(12)}=1.6, \mathrm{p}=0.13$ ), and the

553 scrambled faces (mean \% signal change, US: $0.33 \pm 0.04$, IS: $0.33 \pm 0.04$, paired t-test, $\mathrm{T}_{(12)}=-$

$5540.0026, \mathrm{p}=0.99)$. A repeated-measures ANOVA on percent signal change showed that the 
amplitude of the percent signal change in the V1 ROIs was similar between the scrambled and the

unscrambled pairs (main effect, factor Orientation (upright/inverted): $\mathrm{F}_{(1,12)}=0.028, \mathrm{p}=0.87$,

558 Scrambling: $\left.\mathrm{F}_{(1,12)}=0.87, \mathrm{p}=0.37\right)$.

560 Next, based on the lateralization effects found in behavior and EEG, we analyzed the BOLD

561 contrast responses by hemifield (mean size of the two ROIs from left presentations: $12.69 \pm 0.61$,

562 of the two ROIs from right presentations: $15.19 \pm 1.9$, paired t-test, $\mathrm{T}_{(12)}=-1.7, \mathrm{p}=0.1$ ).

563 Interestingly, in the right hemisphere ROIs (activated by left hemifield presentations), the percent

564 signal change in response to upright faces was significantly higher than to inverted faces for

565 unscrambled faces (mean \% signal change, $\mathrm{U}: 0.35 \pm 0.036, \mathrm{I}: 0.34 \pm 0.04$, paired t-test, $\mathrm{T}_{(12)}=$

$5663.19, \mathrm{p}=0.0077$ (Figure 4C), with 10 out of 13 subjects with a higher percent signal change for

567 the upright face trials) (Figure 4D, left). In contrast, for scrambled faces, responses to upright

568 versus inverted stimuli was similar (mean \% signal change, US: $0.33 \pm 0.036$, IS: $0.34 \pm 0.039$,

569 paired t-test, $\mathrm{T}_{(12)}=-0.8, \mathrm{p}=0.43$, with 6 out of 13 subjects with a higher percent signal change

570 for the upright scrambled face trials (Figure 4D, right)). There also was no significant difference

571 between the response to I and IS $\left(\mathrm{T}_{(12)}=0.63, \mathrm{p}=0.53\right)$, nor between the response to I and US, as

572 predicted $\left(\mathrm{T}_{(12)}=0.17, \mathrm{p}=0.86\right)$. By contrast, when the analysis was restricted to the left

573 hemisphere ROIs (activated by right hemifield presentations), no significant difference between

574 the percent signal change to upright versus inverted faces was found in V1/V2, neither for the

575 unscrambled faces (mean \% signal change, U: $0.32 \pm 0.041, \mathrm{I}: 0.32 \pm 0.043$, paired t-test, $\mathrm{T}_{(12)}=$

$576-0.75, p=0.46$ ), nor for the scrambled faces (mean \% signal change, US: $0.33 \pm 0.045$, IS: 0.32

$577 \pm 0.044$, paired t-test, $\left.\mathrm{T}_{(12)}=0.55, \mathrm{p}=0.58\right)($ Figure $4 \mathrm{E})$. We performed a repeated-measures 
ANOVA on percent signal change, with factors Orientation and Scrambling to check whether there was a difference in the BOLD-signal for U vs. I faces that was specific to the unscrambled faces. This ANOVA, when performed for left-hemifield presentation, confirmed that the response to upright versus inverted faces was different between the scrambled and the unscrambled conditions

582 (main effect, factor Orientation (upright/inverted): $F_{(1,12)}=0.6, p=0.43$, factor Scrambling 583 (scrambled/unscrambled): $\mathrm{F}_{(1,12)}=0.97, \mathrm{p}=0.34$, interaction Orientation $\mathrm{x}$ Scrambling: $\mathrm{F}_{(1,12)}=$ $5844.36, \mathrm{p}=0.05)$. By contrast, for right-hemifield presentation, the response to upright versus 585 inverted faces was similar between the scrambled and the unscrambled conditions (main effect, 586 factor Orientation (upright/inverted): $F_{(1,12)}=1.18, \quad \mathrm{p}=0.29$, factor Scrambling 587 (scrambled/unscrambled): $\mathrm{F}_{(1,12)}=0.014 \mathrm{p}=0.9$, interaction Orientation $\mathrm{x}$ Scrambling: $\mathrm{F}_{(1,12)}=$ $5880.75, \mathrm{p}=0.41)$. This difference between the left versus right presentation paralleled the findings 589 in behavior (Experiments 1 and 2) and EEG (Experiment 3).

591 To avoid saccades, we flashed the stimuli very briefly $(67 \mathrm{~ms})$, a time too short for eye movements 592 to the stimuli of interest since express saccades, the fastest oriented-saccades in humans, peak at $593100 \mathrm{~ms}$ (Fischer \& Ramsperger, 1984). Furthermore, the debriefing showed that subjects were 594 unaware of the presence of faces in the trials. It is therefore unlikely that the difference in the 595 percent signal change between trials with upright and inverted faces (left hemifield presentations, 596 unscrambled faces) reflected differential patterns in eye movements. Additionally, if eye 597 movements toward peripheral items drove this effect, we would expect that the higher the effect 598 size in the BOLD signal was, the worse the accuracy should have been in the central task. Yet, no 599 correlation was found between the effect size in the BOLD signal (computed as the contrast in 600 percent signal change to I vs. U, analysis restricted to left hemifield presentations) and behavior, 
601 both when considering the $\mathrm{d}$ '-measure (Pearson correlation, $\mathrm{r}=0.34, \mathrm{p}=0.25$ ), or when considering the criterion (Pearson correlation, $\mathrm{r}=0.32, \mathrm{p}=0.28$ ).

604 The FFA is known to exhibit a larger BOLD signal in response to faces than to houses (Liu et al., 605 2002). Therefore, we performed a control analysis in the FFA ROIs in order to check whether the 606 differential response in V1/V2 ROIs to upright vs. inverted faces presentations could be due to a 607 differential response to upright versus inverted faces in the FFA, that would feed back to early 608 visual areas. The right FFA could be identified in 8 participants through the contrast face $>$ house 609 (mean coordinates: $(43 \pm 1.41-49 \pm 2.44-20 \pm 2.07)$, mean size: $103.3 \pm 22.81$ voxels).

610 Interestingly, while, in the localizer scans, the FFA ROIs were identified via the higher BOLD611 signal for face presentations relatively to house presentations, in the functional scans that included 612 the flashed peripheral presentation of small faces and houses (Material and Methods for details), 613 no significant difference was observed in the percent signal change between face and house 614 presentation trials (mean percent signal change in face trials: $0.27 \pm 0.025$, in house trials: $0.27 \pm$ 6150.034 , paired t-test, $\left.\mathrm{T}_{(7)}=0.13, \mathrm{p}=0.89\right)$. There was no significant difference either between the 616 percent signal change in face trials split by the hemifield of presentation of the unscrambled faces,

617 to which the FFA is usually responsive (mean percent signal change, unscrambled faces on the 618 left: $0.26 \pm 0.027$ on the right: $0.28 \pm 0.02$, paired t-test, $\mathrm{T}_{(7)}=-0.84 \mathrm{p}=0.42$ ). To test the 619 robustness of these null effects, we ran the same analysis while adding three more participants (n $620=11$ out of 13) for which an FFA-cluster could be identified when using the contrast face $>$ baseline 621 (across the 11 participants, mean Talairach coordinates: $(40.9 \pm 1.58-52.7 \pm 3.41-19.45 \pm 1.77)$, 622 mean size: $93.9 \pm 17.21$ voxels). Adding those three participants did not change the results - no 623 significant difference was found in trials with faces versus houses (mean percent signal change, in 
624 face trials: $0.27 \pm 0.018$, in house trials: $0.28 \pm 0.028$, paired t-test, $\mathrm{T}_{(10)}=-0.38, \mathrm{p}=0.7$ ), and no

625 difference between trials with faces on the left versus on the right hemifield (mean percent signal

626 change, unscrambled faces on the left: $0.27 \pm 0.02$ on the right: $0.28 \pm 0.013$, paired t-test, $\mathrm{T}_{(10)}=$

$627-0.68 \mathrm{p}=0.5)$. Thus, in our experiment, the small, peripherally presented faces did not elicit face-

628 specific activation in the FFA.

629

630 Discussion

631 In the present study, motivated by the remarkably short latency and high localization accuracy of

632 fast saccades to faces, we investigated the hypothesis that the early visual cortex contains selective

633 neural representations for complex objects of high ecological importance, specifically faces.

634 Experiment 1 showed that, compatible with a location in lower visual areas, the specificity of fast

635 saccades to faces is moderate, with "configuration-scrambled" and inverted faces interfering

636 substantially with the localization of upright faces. Experiments 1 and 2 also revealed a key

637 signature of fast saccades to faces, namely an asymmetry between the two hemifields that is

638 characterized by a higher selectivity for upright vs. inverted faces for left hemifield presentations

639 (Experiment 1), spatially more accurate and faster saccades for this hemifield (Experiment 2).

640 Second, an EEG experiment (Experiment 3) revealed that event-related potentials (ERPs)

641 differentiated between upright and inverted faces in the left hemifield from 40 ms post-stimulus

642 onset, i.e., in the latency of the first estimated responses in V1 as measured on the scalp (Foxe et

643 al., 2008). For faces displayed in the right visual hemifield, the earliest significant difference in

644 the ERPs between upright and inverted faces started later, at $98 \mathrm{~ms}$ post-stimulus onset. This

645 asymmetry between the hemifields in the EEG results therefore matched the asymmetry found in 
646 the behavioral experiments. It is worth noting that the EEG difference in latency of the early face-

647 selective response between the left-presentation and right-presentation groups was larger than the

648 difference in minimum saccadic reaction times in Experiment 2. However, participants in that

649 experiment were not only slower for right hemifield presentations but also less accurate. This

650 speed-accuracy tradeoff suggests that behavioral latency differences did not fully compensate

651 neuronal latency differences in Experiment 2. Third, an fMRI experiment (Experiment 4) revealed

652 that in V1/V2, upright faces elicited a higher percent signal change in the BOLD signal than

653 inverted faces. Again, this face-specific response was found specifically for faces displayed in the

654 left visual hemifield, but not for faces displayed in the right visual hemifield. Thus, we found a

655 high degree of agreement between the behavioral, EEG, and fMRI results suggestive of face-

656 selective neuronal populations in early visual cortex.

657

658 Our results come in the wake of recent reports suggestive of face selectivity in early vision. EEG

659 classification results indicate that EEG potentials recorded over occipital locations can predict the

660 location of faces as early as 50ms post-stimulus onset (Martin et al., 2014). In an MEG study, the

661 authors reported, for left (but notably not right) presentations, larger responses to face-like vs.

662 house-like patterns in early visual areas with short latencies (Shigihara \& Zeki, 2014), but the

663 comparison of responses across different channel groupings for different stimuli in that study left

664 open the specificity of the observed effect. A recent ECoG study (Matsuzaki et al., 2015) reported

665 response differences between upright and inverted faces within 40-90ms following stimulus onset

666 over early visual areas V1 and V2. However, most of the electrodes (80\%) were placed in locations

667 corresponding to the upper visual field, therefore resulting in different physical stimulation in the

668 upright face trials versus the inverted face trials. Thus, the differential response might have been 
669 elicited by a difference in the physical stimulation. In Uyar et al., 2015, the authors reported a

670 differential response to faces vs. houses in early visual areas. However, it is problematic to use the

671 face vs. house contrast as a marker for face selectivity in V1 since a differential response to faces

672 versus houses can be driven by low-level differences between the two stimulus classes, given the

673 known selectivity of V1 neurons for low-level features (e.g. luminance, spatial frequency). For

674 this reason, to provide a more selective probe for face selectivity, our study used inverted faces,

675 which have been used as a specific control for face processing in a large number of studies (Haxby

676 et al., 1999 ; Itier \& Taylor, 2002 ; Kanwisher et al., 1998). As a further test, we created scrambled

677 faces that are unrecognizable as faces yet preserve the original faces' low-level properties; we

678 found that unscrambled inverted faces and scrambled faces in their upright and inverted version

679 elicited a similar percent BOLD signal change in early visual areas ROIs, lower than the signal

680 elicited by unscrambled upright faces. This strongly confirms that the response in the early visual

681 cortex that we found is a response specific to faces as an abstract category, that cannot be accounted

682 for by low-level properties of faces.

683

684 The left / right asymmetry that we found across the experiments parallels the well-known left

685 hemifield advantage found for face recognition and categorization (Gilbert \& Bakan, 1973,

686 Rhodes, 1985, Sergent \& Bindra, 1981) and the right hemisphere advantage for neural

687 representation of faces (Yovel et al., 2008). It has been postulated that asymmetries in visual

688 processing between the left and right hemisphere relate to the level of processing (local vs. global),

689 spatial frequency content of stimuli, or the visual pathway (magno- vs. parvocellular) (for a review,

690 see (Hellige, 1996)). While the mechanisms responsible for the face processing asymmetry are

691 beyond the scope of this paper, it might be of significant interest for future studies to directly 
692 investigate the relationship between the low-level face processing asymmetry with the asymmetry

693 of high-level face processing (e.g., in the FFA).

694

696 Our study suggests the intriguing hypothesis that there might be neural representations selective

697 for certain complex object classes (such as faces) at the lowest levels of the visual hierarchy. This

698 finding challenges the standard simple-to-complex hierarchical description of the visual system

699 according to which complex object categories are processed at high levels of the visual hierarchy.

700 It is unlikely that the selectivity in early visual areas for upright faces identified in our study

701 (Experiment 4) is the result of feedback activity from areas belonging to the core network for face

702 perception, such as the FFA or the OFA (occipital face area) (Haxby et al., 2000). First, in our

703 study, the usual contrast of Faces vs. Houses, to which the FFA is normally responsive (Liu et al.,

704 2002), did not reveal any significant difference of the percent BOLD signal. This might be due to

705 the fact that the faces were presented in the periphery while the FFA is mostly responsive to central

706 face presentations (Levy et al., 2001). Second, the debriefing conducted at the end of Experiment

7074 indicated that subjects did not notice the presence of faces or houses. Those subjective reports

708 suggest that the masking was efficient, thus preventing feedback-driven face-specific activity in

709 V1/V2 (Fahrenfort et al., 2007). Taken together, the early latency of the face-specific response

710 found in the EEG signals and the localization of the face-specific response in V1/V2 suggest that

711 face-specific responses emerge during a first forward sweep of processing and truly reflect a

712 selectivity to faces in early vision rather than the propagation in feedback of the activity from

713 higher-level areas. 
715 Given its position at a low level of the visual processing hierarchy, we would not expect that the

716 degree of face selectivity in V1/V2 would be comparable to the high degree of face selectivity in

717 the FFA, which has been shown to be involved in the discrimination of different faces identities

718 (for a review, see Kanwisher et al., 2006). In fact, the observation that the fast saccades were

719 attracted toward the configuration-scrambled faces (Experiment 1) suggests that face

720 representations in V1/V2 are not selective for the configuration of the faces, in line with findings

721 about face representations in higher areas of occipital cortex that are not selective for the face-part

722 configurations (the Occipital Face Area, OFA) (Pitcher et al., 2007; Liu et al 2010), in contrast to

723 face representations further downstream in fusiform cortex (Andrews et al., 2010; Liu et al., 2010).

725 The face-specific neural response that we observed in V1/V2, starting at $40 \mathrm{~ms}$ post-stimulus onset, 726 constitutes a likely underpinning for the fast saccadic detection of faces. Such a model of

727 "shortcuts" in hierarchical visual processing, with early visual representations directly providing 728 input to decision circuits for fast motor responses is compatible with recent theories of

729 thalamocortical processing (Sherman, 2016) in which corticofugal projections from layer 5 in early

730 visual areas could carry task-relevant signals directly to the superior colliculus. It will be very

731 interesting in future work to explore the plasticity of this circuit, to see whether it is possible to

732 learn fast saccades for new object classes in addition to faces by effecting object-specific plasticity

733 in early visual areas. 


\section{Author contribution}

736 F.C, M.R., X.J., J.G.M., S.J.T designed experiments. F.C. ran experiments with assistance from

737 L.B. F.C. and M.R. analyzed the data. All co-authors contributed to the writing of the manuscript. 


\section{References}

740 Aine, C. J., Supek, S., George, J. S., Ranken, D., Lewine, J., Sanders, J., \& Wood, C. C. (1996).

741 Retinotopic organization of human visual cortex: departures from the classical model. Cerebral 742 Cortex, 6(3), 354-361.

743 Andrews, T.J., Davies-Thompson, J., Kingstone, A., and Young, A.W. (2010). Internal and

744 External Features of the Face Are Represented Holistically in Face-Selective Regions of Visual

745 Cortex. J. Neurosci., 30(9), 3544-3552.

746 Beck, D.M., and Kastner, S. (2005). Stimulus context modulates competition in human extrastriate

747 cortex. Nat. Neurosci., 8(8), 1110-1116.

748 Brett, M., Anton, J. L., Valabregue, R., \& Poline, J. B. (2002). Region of interest analysis using

749 an SPM toolbox. 8th international conference on functional mapping of the human brain (Vol.

750 16, No. 2, p. 497).

751

752 Brilhault, A., Mathey, M., Jolmes, N., \& Thorpe, S. J. (2011). Measuring the receptive field sizes

753 of the mechanisms underlying ultra-rapid saccades to faces. Perception ECVP abstract, 40, 157-

754157.

755

756 Cauchoix, M., Barragan-Jason, G., Serre, T., \& Barbeau, E. J. (2014). The neural dynamics of

757 face detection in the wild revealed by MVPA. J. Neurosci., 34(3), 846-854.

758 Crouzet, S.M., Thorpe, S.J. (2010). Fast saccades toward faces : Face detection in just $100 \mathrm{~ms} . J$

759 Vis., 10(4), 1-17. 
760 Crouzet, S. M., \& Thorpe, S. J. (2011). Low-level cues and fast face detection. Frontiers in

761 psychology, 2, 342.

762 Delorme, A., \& Makeig, S. (2004). EEGLAB: an open source toolbox for analysis of single-trial

763 EEG dynamics including independent component analysis. J. Neurosci. Methods, 134(1), 9-21.

764 Di Russo et al. (2005). Identification of the neural sources of the pattern-reversal

765 VEP. NeuroImage, 24(3), 874-886.

766 Dougherty, R. F., Koch, V. M., Brewer, A. A., Fischer, B., Modersitzki, J., \& Wandell, B. A.

767 (2003). Visual field representations and locations of visual areas V1/2/3 in human visual

768 cortex. J. Vis., 3(10), 1-1.

769 Dumoulin, S.O., Wandell, B.A. (2008). Population receptive field estimates in human visual 770 cortex. Neuroimage, 39(2), 647-660.

771 Eimer, M., Gosling, A., Nicholas, S., \& Kiss, M. (2011). The N170 component and its links to

772 configural face processing: a rapid neural adaptation study. Brain research, 1376, 76-87.

773 Engel, S. A., Glover, G. H., \& Wandell, B. A. (1997). Retinotopic organization in human visual

774 cortex and the spatial precision of functional MRI. Cerebral cortex, 7(2), 181-192.

775

776 Fabre-Thorpe, M., Delorme, A., Marlot, C., \& Thorpe, S. (2003). A limit to the speed of processing

777 in ultra-rapid visual categorization of novel natural scenes. J. Cogn. Neurosci., 13(2), 171-180.

778

779 Fahrenfort, J. J., Scholte, H. S., \& Lamme, V. A. (2007). Masking disrupts reentrant processing

780 in human visual cortex. J. Cogn. Neurosci., 19(9), 1488-1497. 
781 Fischer, B., Ramsperger, E. (1984). Experimental Brain Research Human express saccades:

782 extremely short reaction times of goal directed eye movements. Exp. Brain. Res., 57, 191-195.

783 Fischer, B., Weber, H. (1993). Express saccades and visual attention. Behav. Brain Sci., 16(3),

$784 \quad 553-567$.

785 Foxe, J.J., Simpson, G. (2002). Flow of activation from V1 to frontal cortex in humans. Exp. Brain

786 Res., $142(1), 139-150$.

787 Foxe, J.J., Strugstad, E.C., Sehatpour, P., Molholm, S., Pasieka, W., Schroeder, C.E., McCourt, 788 M.E. (2008). Parvocellular and magnocellular contributions to the initial generators of the visual 789 evoked potential: High-density electrical mapping of the "C1" component. Brain Topogr., 21(1), $790 \quad 11-21$

791 Gilbert, C., \& Bakan, P. (1973). Visual asymmetry in perception of

792 faces. Neuropsychologia, 11(3), 355-362.

793 Glezer, L.S., Kim, J., Rule, J., Jiang, X., and Riesenhuber, M. (2015). Adding Words to the Brain's

794 Visual Dictionary: Novel Word Learning Selectively Sharpens Orthographic Representations in

795 the VWFA. J. Neurosci., 35(12), 4965-4972.

796 Grill-Spector, K., Knouf, N., and Kanwisher, N. (2004). The fusiform face area subserves face 797 perception, not generic within-category identification. Nat. Neurosci., 7(5), 555-562.

798 Guyader, N., Chauvin, A., Boucart, M., \& Peyrin, C. (2017). Do low spatial frequencies explain

799 the extremely fast saccades towards human faces? Vis. Res., 133, 100-111.

800 Haxby, J. V., Ungerleider, L.G., Clark, V.P., Schouten, J.L., Hoffman, E.A., and Martin, A. (1999).

801 The effect of face inversion on activity in human neural systems for face and object perception.

802 Neuron, 22(1), 189-199. 
803 Haxby, J., Hoffman, E., Gobbini, M. (2000). The distributed human neural system for face

804 perception. Trends Cogn. Sci., 4(6), 223-233.

805 Heeman, Jessica, Stefan Van der Stigchel, and Jan Theeuwes. The influence of distractors on

806 express saccades. J. Vis., 17.1 (2017): 35-35.

807

808 Hellige, J. B. (1996). Hemispheric asymmetry for visual information processing. Acta

809 Neurobiologiae Experimentalis, 56, 485-497.

810 Honey, C., Kirchner, H., Vanrullen, R. (2008). Faces in the cloud : Fourier power spectrum biases

811 ultrarapid face detection. J. Vis., 8(12), 1-13.

812 Hubel, D. H., \& Wiesel, T. N. (1962). Receptive fields, binocular interaction and functional

813 architecture in the cat's visual cortex. The Journal of physiology, 160(1), 106-154.

814 Itier, R.J., Taylor, M.J. (2002). Inversion and contrast polarity reversal affect both encoding and

815 recognition processes of unfamiliar faces: a repetition study using ERPs. Neuroimage, 15(2), 353-

816372.

817 Itier, R.J., Alain, C., Sedore, K., McIntosh, A.R. (2007). Early face processing specificity: it's in

818 the eyes! J. Cogn. Neurosci,. 19(11), 1815-1826.

819 Jiang, X., Rosen, E., Zeffiro, T., Vanmeter, J., Blanz, V., Riesenhuber, M. (2006). Evaluation of a

820 shape-based model of human face discrimination using FMRI and behavioral techniques. Neuron,

$82150(1), 159-172$.

822 Kanwisher, N., Mcdermott, J., Chun, M.M. (1997). The Fusiform Face Area : A Module in Human

823 Extrastriate Cortex Specialized for Face Perception. J. Neurosci., 17(11), 4302-4311. 
824 Kanwisher, N., Tong, F., Nakayama, K. (1998). The effect of face inversion on the human fusiform

825 face area. Cognition, 68(1), B1-B11.

826 Kanwisher, N., \& Yovel, G. (2006). The fusiform face area: a cortical region specialized for the

827 perception of faces. Philosophical Transactions of the Royal Society of London B: Biological

$828 \quad$ Sciences, 361(1476), 2109-2128.

829 Kirchner, H., and Thorpe, S.J. (2006). Ultra-rapid object detection with saccadic eye movements:

830 Visual processing speed revisited. Vis. Res., 46(11), 1762-1776.

831 Levine, S. C., Banich, M. T., \& Koch-Weser, M. P. (1988). Face recognition: A general or specific

832 rig specific right hemisphere capacity? Brain and Cognition, 8(3), 303-325.

833 Levy, I., Hasson, U., Avidan, G., Hendler, T., Malach, R. (2001). Center-periphery organization

834 of human object areas. Nat. Neurosci. 4(5), 533-539.

835 Liu, J., Harris, A., Kanwisher, N. (2002). Stages of processing in face perception: an MEG study.

836 Nat. Neurosci., 5(9), 910-916.

837 Liu, J., Harris, A., and Kanwisher, N. (2010). Perception of face parts and face configurations: an

838 FMRI study. J. Cogn. Neurosci., 22(1), 203-211.

839 Macknik, S.L., Livingstone, M.S. (1998). Neuronal correlates of visibility and invisibility in the 840 primate visual system. Nat. Neuro., 1(2), 144-149.

841 Macmillan, N. A., \& Creelman, C. D. (2005). Detection Theory: A User's Guide Lawrence

842 Erlbaum Associates. New York, 73.

844 Martin, J., Riesenhuber, M., Thorpe, S. (2014). The time-course of face-selective ERP activation 845 during ultra-rapid saccades. J. Vis., 14(10), 134-134. 
846 Matsuzaki, N., Schwarzlose, R.F., Nishida, M., Ofen, N., Asano, E. (2015). Upright face-

847 preferential high-gamma responses in lower-order visual areas: Evidence from intracranial

848 recordings in children. Neuroimage, 109, 249-259.

849 Maunsell, J. H., \& Newsome, W. T. (1987). Visual processing in monkey extrastriate

850 cortex. Annual review of neuroscience, 10(1), 363-401.

852 Miller, J., Beutinger, D., \& Ulrich, R. (2009). Visuospatial attention and redundancy gain. Psych.

853 Research, 73(2), 254-262.

855 Mooshagian, E., Kaplan, J., Zaidel, E., \& Iacoboni, M. (2008). Fast visuomotor processing of

856 redundant targets: the role of the right temporo-parietal junction. PLoS One, 3(6), e2348.

857 Motter, B.C. (2009). Central V4 receptive fields are scaled by the V1 cortical magnification and

858 correspond to a constant-sized sampling of the V1 surface. J. Neurosci., 29(18), 5749-5757.

859 Oostenveld, R., Fries, P., Maris, E., Schoffelen, J.M. (2011). FieldTrip: Open source software for

860 advanced analysis of MEG, EEG, and invasive electrophysiological data. Comput. Intell.

861 Neurosci., 2011.

862 Oosterhof, N.N., Todorov, A. (2008). The functional basis of face evaluation. Proc. Natl. Acad.

863 Sci., 105(32), 11087-11092.

864 Perrinet, L. U., Adams, R. A., \& Friston, K. J. (2014). Active inference, eye movements and

865 oculomotor delays. Biol. Cybernetics, 108(6), 777-801.

866 Pitcher, D., Walsh, V., Yovel, G., Duchaine, B. (2007). TMS evidence for the involvement of the

867 right occipital face area in early face processing. Curr. Biol., 17(18), 1568-1573. 
868 Pizzagalli, D., Regard, M., \& Lehmann, D. (1999). Rapid emotional face processing in the

869 human right and left brain hemispheres: an ERP study. Neuroreport, 10(13), 2691-2698.

870 Poncet, M., Reddy, L., Fabre-thorpe, M. (2012). A need for more information uptake but not

871 focused attention to access basic-level representations. J. Vis., 12(1), 1-16.

872 Prieto, E.A., Caharel, S., Henson, R., Rossion, B. (2011). Early (n170/m170) face-sensitivity

873 despite right lateral occipital brain damage in acquired prosopagnosia. Front. Hum. Neurosci., 5,

874138.

875 Kalesnykas, R.P., Hallett, PE (1987). The differentiation of visually guided and anticipatory

876 saccades in gap and overlap paradigms. Exp. Brain Res., 68(1), 115-121.

877 Riesenhuber, M., \& Poggio, T. (2002). Neural mechanisms of object recognition. Current

878 opinion in neurobiology, 12(2), 162-168.

879

880 Rhodes, G. (1985). Lateralized processes in face recognition. British journal of

881 Psychology, 76(2), 249-271.

882 Rossion, B., Caharel, S. (2011). ERP evidence for the speed of face categorization in the human

883 brain: Disentangling the contribution of low-level visual cues from face perception. Vision Res.,

$88451(12), 1 \Omega 97-1311$.

885 Rousselet, G. A., Husk, J. S., Bennett, P. J., \& Sekuler, A. B. (2005). Spatial scaling factors

886 explain eccentricity effects on face ERPs. J. Vis., 5(10), 1-1.

887 Rousselet, G.A. (2012). Does filtering preclude us from studying ERP time-courses? Front.

888 Psychol., 3, 1-9. 
889 Scholl, C.A., Jiang, X., Martin, J.G., Riesenhuber, M. (2014). Time Course of Shape and Category

890 Selectivity Revealed by EEG Rapid Adaptation. J. Cogn. Neurosci., 26(2), 408-421.

891 Schwarz, W. (1996). Further tests of the interactive race model of divided attention: the effects

892 of negative bias and varying stimulus — onset asynchronies. Psychol. Res., 58(4), 233-245.

893

894 Sergent, J., \& Bindra, D. (1981). Differential hemispheric processing of faces: Methodological 895 considerations and reinterpretation. Psych. Bulletin, 89(3), 541-554.

896 Sherman, S.M. (2016). Thalamus plays a central role in ongoing cortical functioning. Nat.

897 Neurosci., 19(4), 533-541.

898 Shigihara, Y., Zeki, S. (2014). Parallel processing of face and house stimuli by V1 and specialized 899 visual areas: a magnetoencephalographic (MEG) study. Front. Hum. Neurosci., 8, 901.

900 Stojanoski, B., Cusack, R. (2014). Time to wave good-bye to phase scrambling: creating controlled 901 scrambled images using diffeomorphic transformations. J. Vis., 14(12), 1-16.

902 Thorpe, S., Fize, D., Marlot, C. (1996). Speed of processing in the human visual system. Nature, $903 \quad 381,520-522$.

904 Turatto, M., \& Betta, E. (2006). Redundant visual signals boost saccade execution. Psych.

905 Bulletin \& Review, 13(5), 928-932.

906 Uyar, F., Shomstein, S., Greenberg, A.S., Behrmann, M. (2015). Retinotopic information interacts

907 with category selectivity in human ventral cortex. Neuropsychologia, 92, 90-106.

908 VanRullen, R., Thorpe, S.J. (2001). The time course of visual processing: from early perception 909 to decision-making. J. Cogn. Neurosci., 13(4), 454-461. 
910 Vogels, R., \& Orban, G. A. (1996). Coding of stimulus invariances by inferior temporal neurons.

911 In Prog. In Brain Research, 112, 195-211.

912 Xu, Y., Liu, J., \& Kanwisher, N. (2005). The M170 is selective for faces, not for

913 expertise. Neuropsychologia, 43(4), 588-597.

914 Young, A. W., Hay, D. C., \& McWeeny, K. H. (1985). Right cerebral hemisphere superiority for

915 constructing facial representations. Neuropsychologia, 23(2), 195-202.

916 Yovel, G., Tambini, A., \& Brandman, T. (2008). The asymmetry of the fusiform face area is a

917 stable individual characteristic that underlies the left-visual-field superiority for

918 faces. Neuropsychologia, 46(13), 3061-3068. 
bioRxiv preprint doi: https://doi.org/10.1101/2020.03.14.987735; this version posted March 15,2020 . The copyright holder for this preprint (which was not certified by peer review) is the author/funder, who has granted bioRxiv a license to display the preprint in perpetuity. It is made available under aCC-BY-ND 4.0 International license.

A.

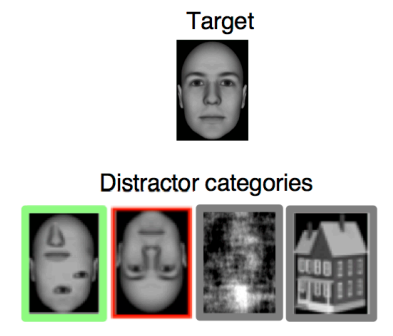

B.

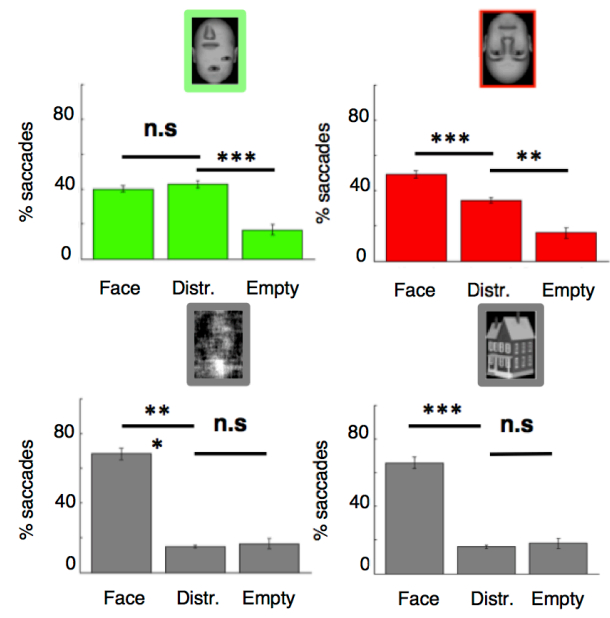

Figure 1

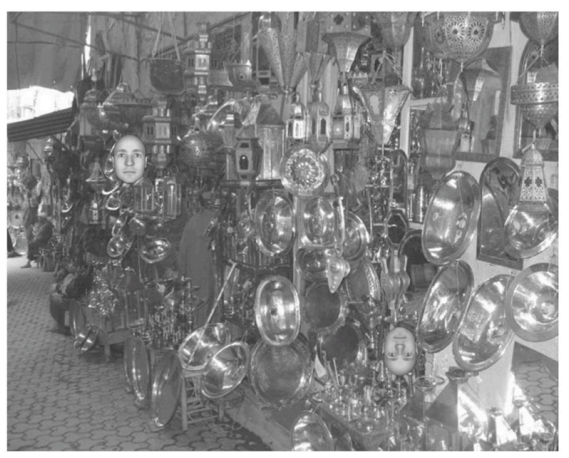

C.
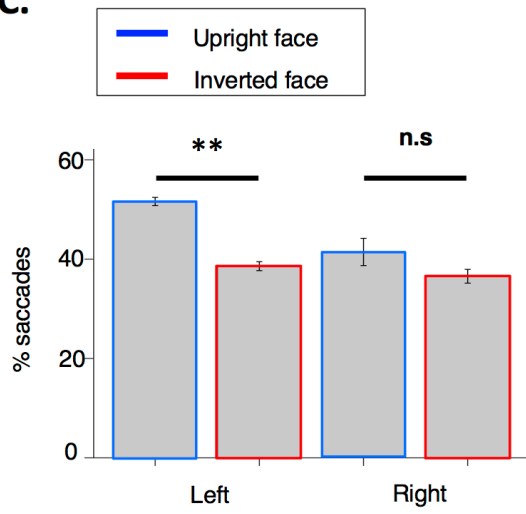
bioRxiv preprint doi: https://doi.org/10.1101/2020.03.14.987735; this version posted March 15, 2020. The copyright holder for this preprint (which was not certified by peer review) is the author/funder, who has granted bioRxiv a license to display the preprint in perpetuity. It is made available under aCC-BY-ND 4.0 International license.

\section{Figure 2}

A.

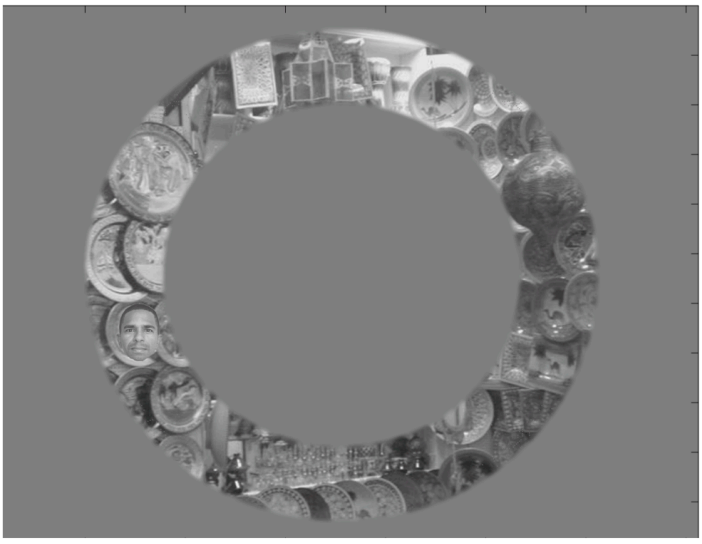

C.

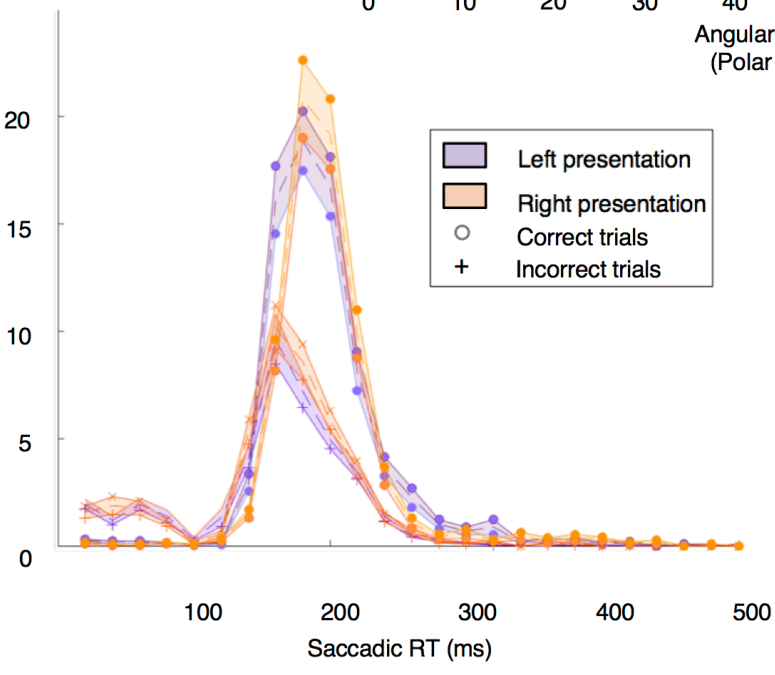


Figure 3

A.

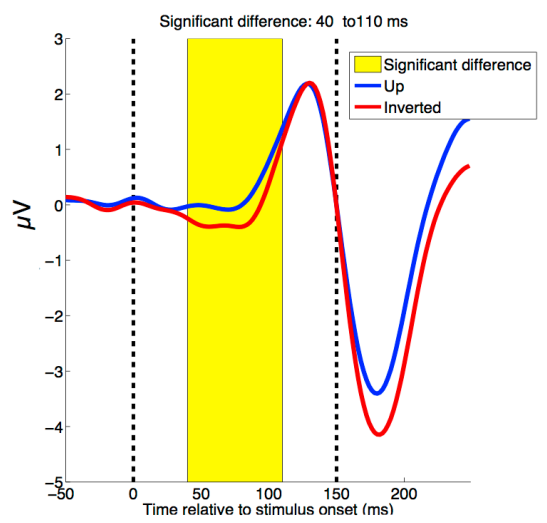

C.

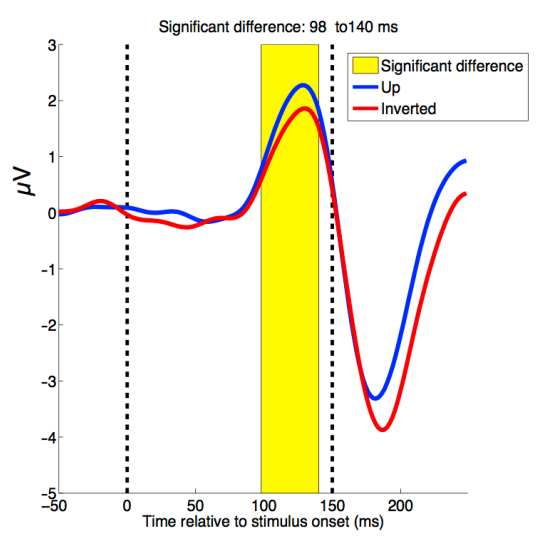

B.

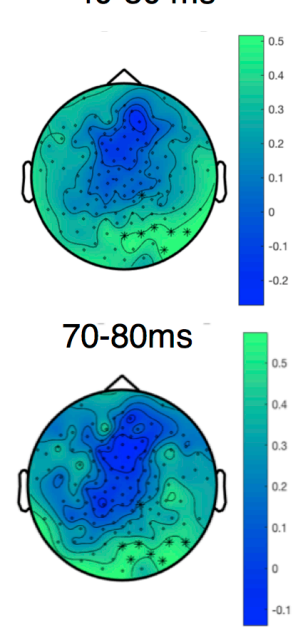

D.

$100-110 \mathrm{~ms}$

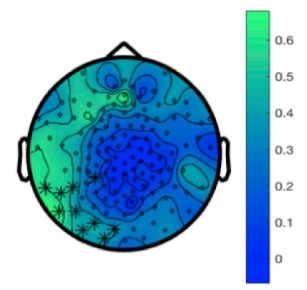

$130-140 \mathrm{~ms}$

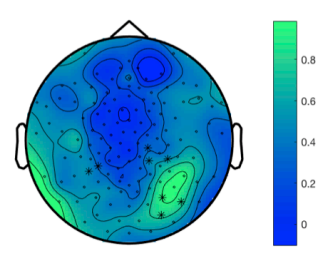

$50-60 \mathrm{~ms}$

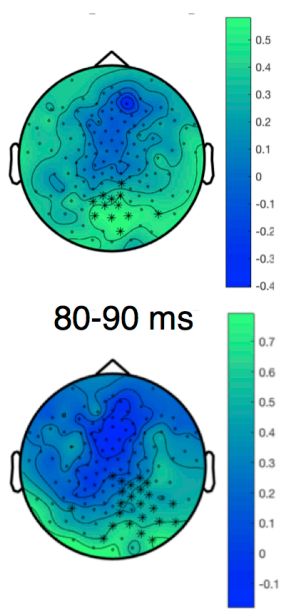

$110-120 \mathrm{~ms}$

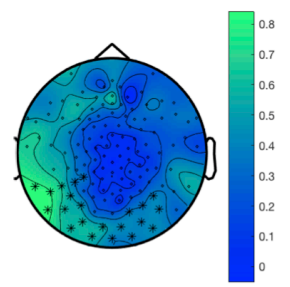

$60-70 \mathrm{~ms}$

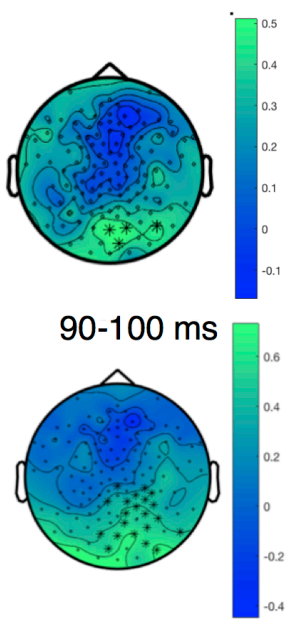

$120-130 \mathrm{~ms}$

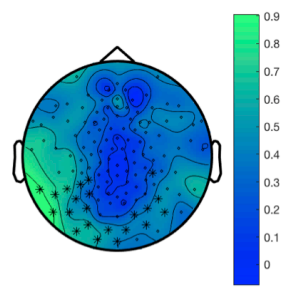


bioRxiv preprint doi: https://doi.org/10.1101/2020.03.14.987735; this version posted March 15, 2020. The copyright holder for this preprint (which was not certified by peer review) is the author/funder, who has granted bioRxiv a license to display the preprint in perpetuity. It is made available under aCC-BY-ND 4.0 International license.

A.

Figure 4

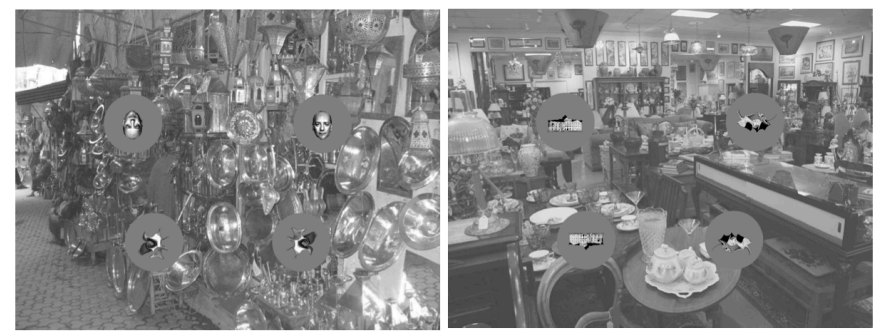

B.

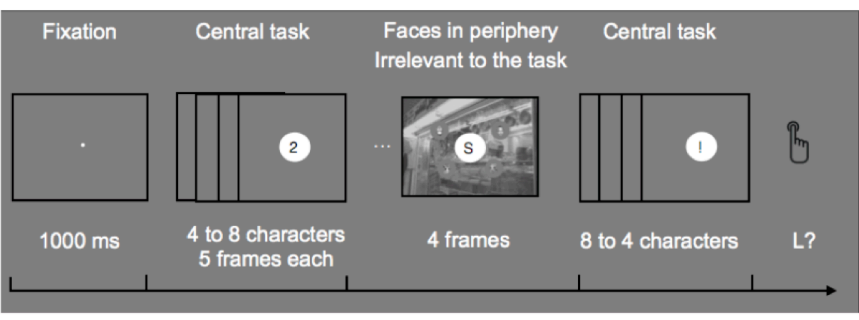

C.
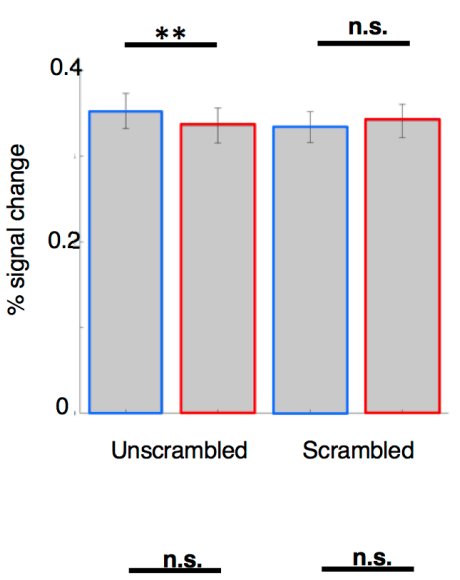

\section{E.}

Upright face Inverted face

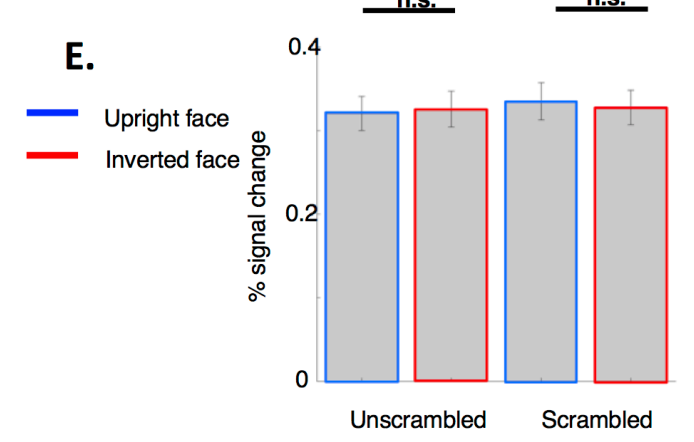

D.

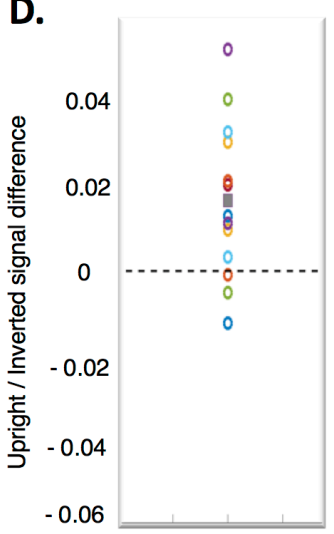

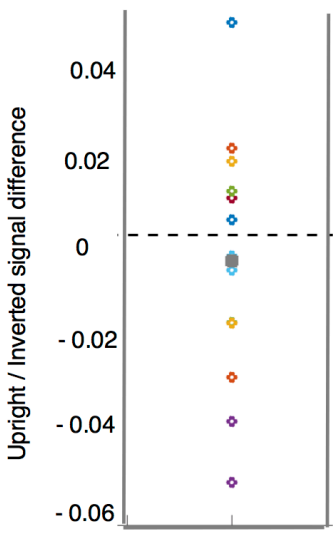




\section{Figure Legends}

923 Figure 1. Experiment 1: Fast saccades toward faces have limited selectivity. A. Stimuli and

924 paradigm. At each trial, one face and a distractor (from left to right: a configuration-scrambled

925 face, an inverted face, a phase-scrambled face, a house) were displayed at 2 positions, here Left

926 up and Right bottom among 4 positions (Left up, Right up, Left bottom, Right bottom). B. Percent

927 of first saccades landing in the quadrant with the face, with the distractor, or toward one of the

928 empty quadrants. Results for configuration-scrambled faces are shown in green, for inverted faces

929 in red, for phase-scrambled faces in gray (left), for houses in gray (right)). In the configuration-

930 scrambled face condition, saccades were oriented as often toward the configuration-scrambled face

931 as toward the target (the upright face). When the distractor was an inverted face, saccades were

932 directed more often toward the target than toward the inverted face distractor. In the control

933 conditions where distractors were houses and phase-scrambled faces, the percent of saccades

934 oriented toward the distractor was similar to the percentage of saccades toward an empty quadrant.

935 C. Percent of first saccades in the inverted face condition when the target and the distractor were

936 either both in the left hemifield, or both in the right hemifield. When they were in the left hemifield,

937 saccades were more often directed toward the target than toward the distractor. When they were

938 in the right hemifield, saccades were as often oriented toward the distractor as toward the target.

$939\left(* * * \mathrm{p}<0.001, * * \mathrm{p}<0.005,{ }^{*} \mathrm{p}<0.05\right)$. Data are presented as mean $\pm \mathrm{SEM}$.

941 Figure 2. Experiment 2: Faces are saccaded to faster when they are in the left hemifield. A.

942 Example stimulus. The face target could be pasted anywhere within an annulized natural scene. B.

943 Spatial precision of target saccades in Experiment 2. The plot shows the percentage of saccades 
944 landing within an angular window centered on the face, averaged across participants, as a function

945 of the size of the window (degrees of polar angle). The slope rises steeply up to 15 degrees, as

946 progressively more face target-directed saccades are included, then becomes shallower with

947 constant slope, presumably since further increases in window size include progressively more

948 saccades targeted at distractors. The total size of the window is twice the value indicated in abscissa

949 since it extends to both sides. This graph thus reveals that most of the saccades aggregated in an

950 area centered on the face and expanding at \pm 15 degrees of polar angle. C. Distribution of saccadic

951 reaction times for correct and incorrect saccades, for left-presentation and right-presentation of the

952 faces, averaged across participants. The distribution of correct saccades diverges from the

953 distribution of incorrect saccades earlier for left than for right presentation. This shows that

954 saccades start to be selective earlier for left than for right presentations.

Figure 3. Experiment 3: EEG data reveal significant face-specific responses starting at $40 \mathrm{~ms}$

957 post-stimulus onset for left-stimulus presentations. Event-related potentials (ERPs) computed

958 within the cluster found in the time window [0-150] ms. Left-presentation group. A. Mean ERPs

959 elicited by upright versus inverted faces, computed across the channels belonging to the cluster.

960 The cluster ranges between [40-110] ms post-stimulus. The time window over which the clustering

961 analysis was run is indicated by dashed lines, the window of significance is in yellow. B. Time

962 course of the cluster, electrodes significant during the whole time window (10 ms bins) are

963 indicated by stars: the cluster is initially lateralized in the right hemisphere. Right-presentation

964 group. C. Mean ERPs elicited by upright versus inverted faces, computed across the channels

965 belonging to the cluster. The cluster ranges between [98-140] ms post-stimulus D. Time course

966 of the cluster, electrodes significant during the whole time window (10 ms bins) are indicated by 
967 stars: the cluster is initially lateralized in the left hemisphere. It becomes more spread-out starting around 110-120 ms poststimulus onset.

Figure 4. Experiment 4: fMRI data provide evidence for face-specific responses in V1/V2.

971 A. Example stimuli. Left: example of a face trial, one face is pasted in its 4 different versions

972 (inverted, upright, scrambled, and inverted scrambled on the top left, top right, bottom left and

973 bottom right respectively) in the middle of gray circles. Right: example of a house-trial, built in

974 the same way as the face-trial. B. Experimental design. Participants performed a central letter

975 detection task in a rapid visual serial stream. In this stream, a natural image with faces (or houses)

976 at $7^{\circ}$ eccentricity was displayed at an unpredictable moment. Subjects were not told about the

977 presence of faces/houses. C. Percent signal change within V1/V2 ROIs averaged within stimulus

978 category across the ROIs activated by left visual presentations (top left and bottom left). There is

979 a significantly higher percent signal change in trials with upright (unscrambled) faces relatively to

980 trials with inverted (unscrambled) faces, but no significant difference for the comparison upright

981 (scrambled) faces versus inverted (scrambled faces). D. Left. Representation of the difference

982 between the percent signal change evoked by the upright (unscrambled) faces and by the inverted

983 (unscrambled) faces, for left-hemifield presentations. Right. Representation of the difference

984 between the percent signal change evoked by the upright (scrambled) faces and by the inverted

985 (scrambled) faces, for left-hemifield presentations. Each dot corresponds to one participant. The

986 average value of those points is represented by a gray, filled, square. E. Percent signal change

987 averaged within stimulus category across the ROIs activated by right visual presentations (top right

988 and bottom right). The percent signal change elicited by upright and inverted faces in this visual

989 hemifield is not significantly different, both for unscrambled and scrambled faces. 